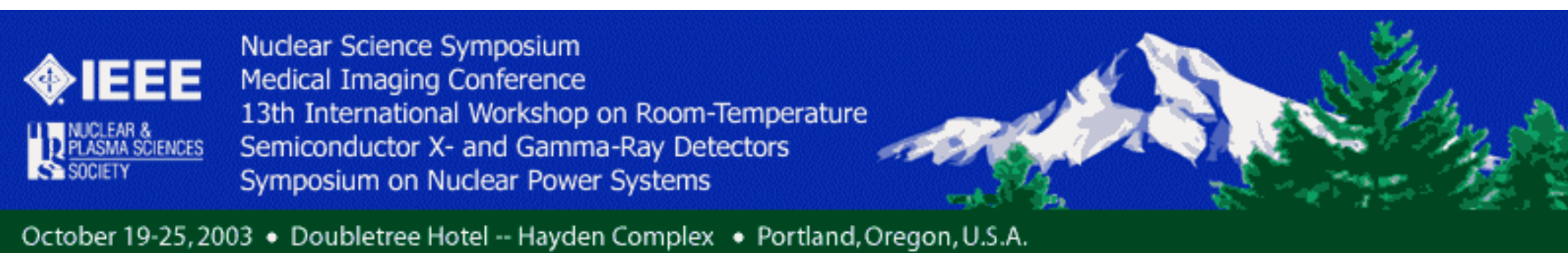

\title{
Performance Study of New Pixel
}

\section{Hybrid Photon Detector Prototypes}

for the LHCb RICH counters

Matthias Moritz

(on behalf of the LHCb Pixel Group)

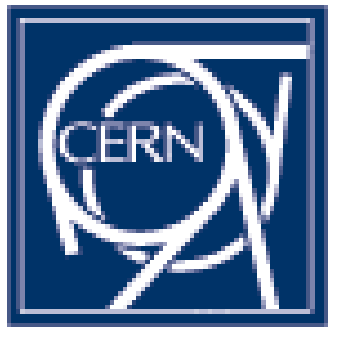

-Detector description

-Performance tests

-Summary

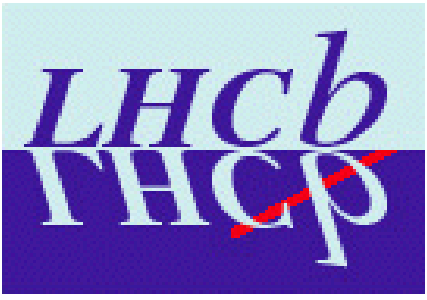




\section{$\underline{\text { Performance Tests }}$}

Real pixel size $62.5 \times 500 \mu \mathrm{m}^{2}$ :

$1024 \rightarrow 8192$ pixels

- two $40 \mathrm{MHz}$ HPD prototypes tested with $98.5 \%$ and $95.6 \%$ working bonds (out of 8192 ) (one $10 \mathrm{MHz}$ HPD prototype with $>\mathbf{9 9 . 9 \%}$ working bonds)
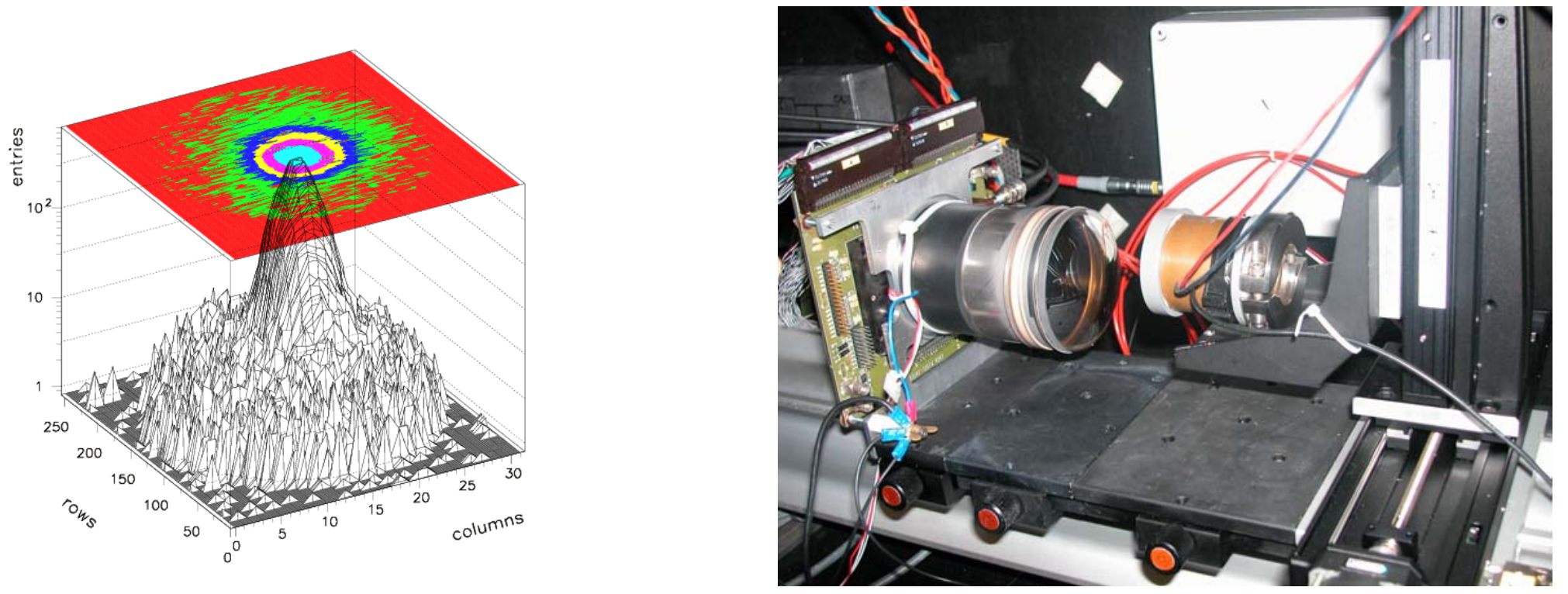

fast, pulsed LED used as light source \& some test beam data 


\section{Discrimination Threshold}

Analogue front end consists of:

charge preamplifier, shaper and discriminator

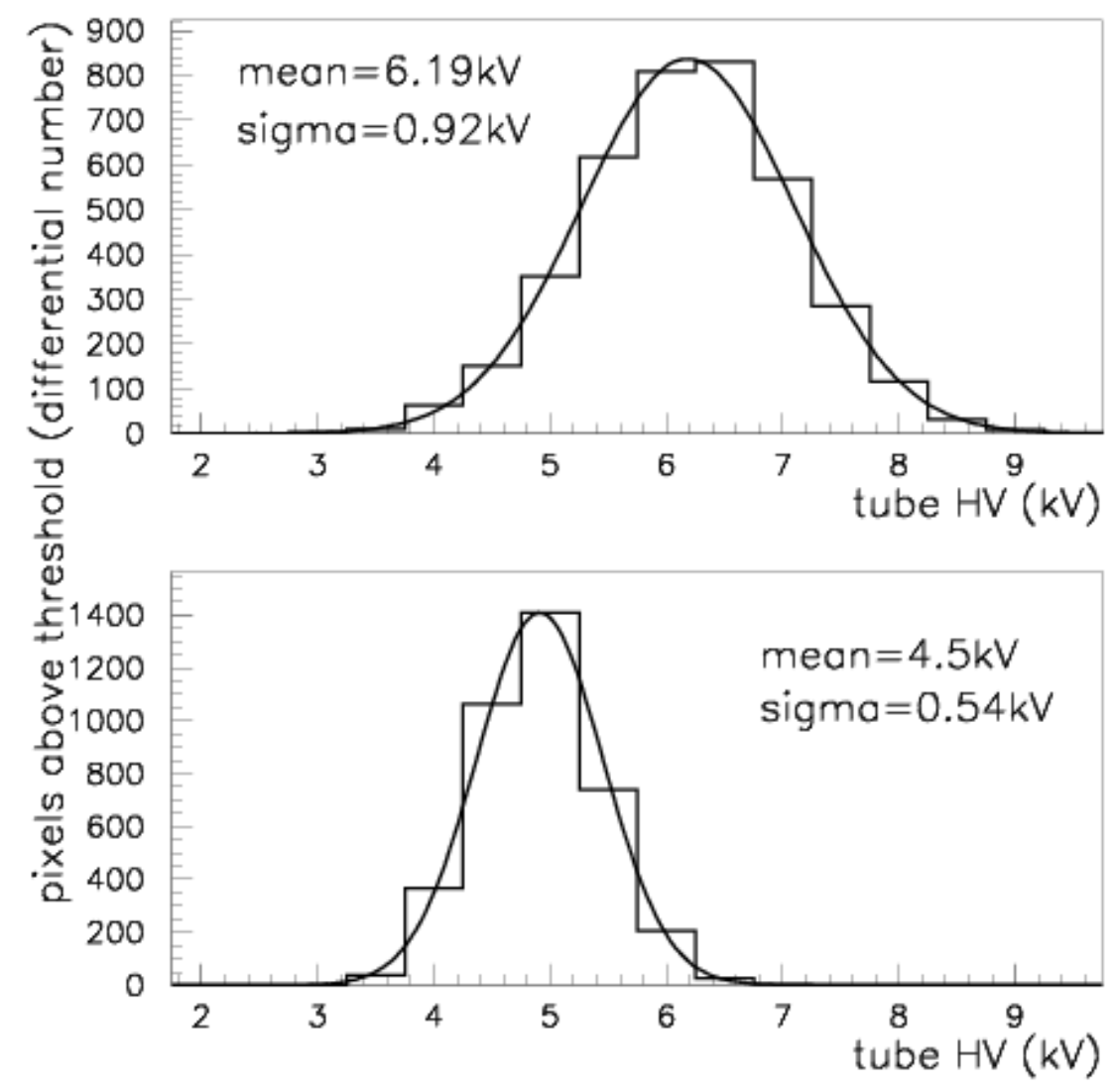

- discriminating threshold can be adjusted

- threshold ( 1700e $)$ and its spread $\left(\sim 250 \mathrm{e}^{-}\right)$within specifications

- 3 bit pixel adjustment to achieve lower and narrower threshold distribution

$>$ efficiency improvement at $20 \mathrm{kV}: \sim 1 \%$ 


\section{Detector Bias \&Tube High Voltage}
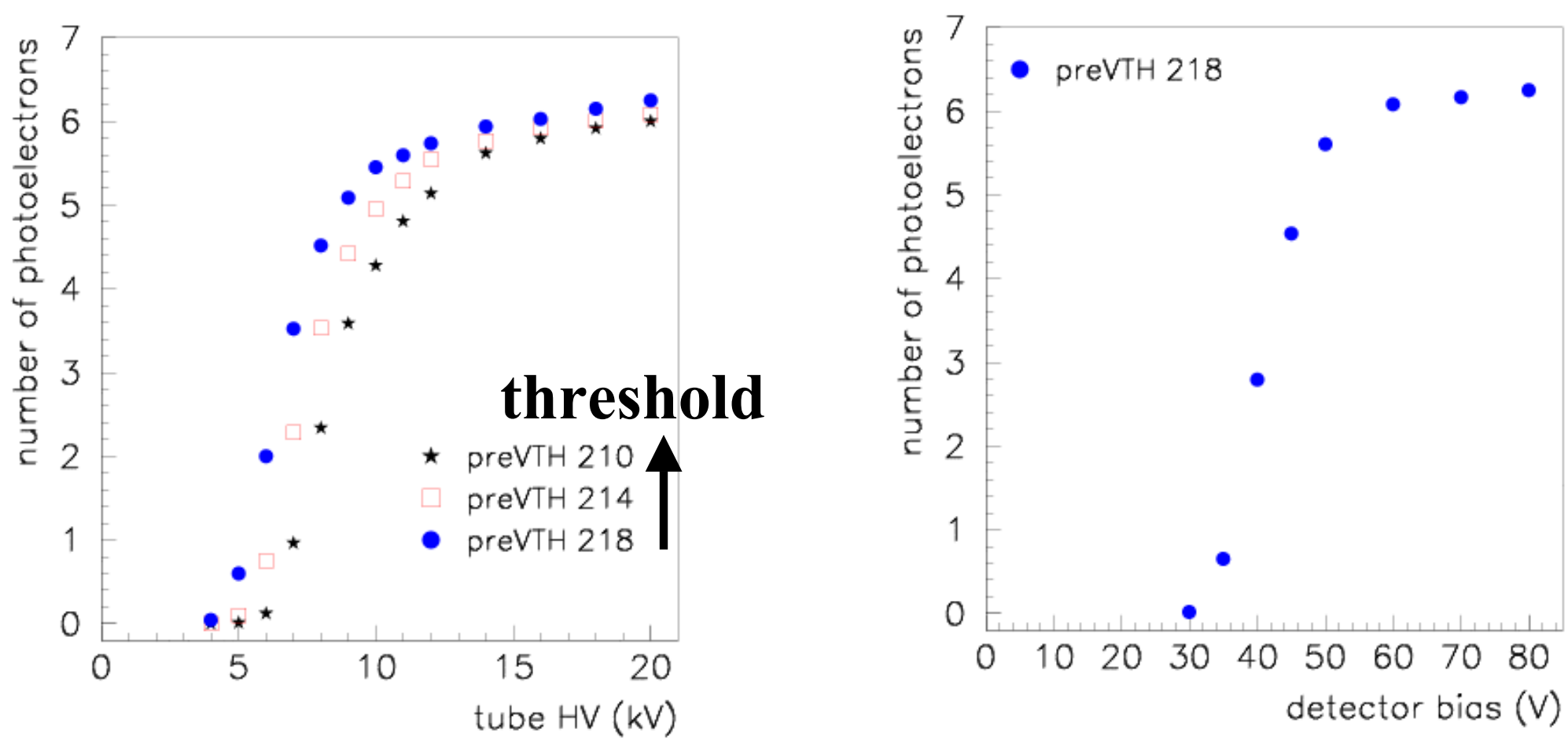

-Performance vs. tube $\mathrm{HV}$ and detector bias studied

$>$ detector has to be over-depleted to collect the charge efficiently 


\section{Detection Efficiency Results}

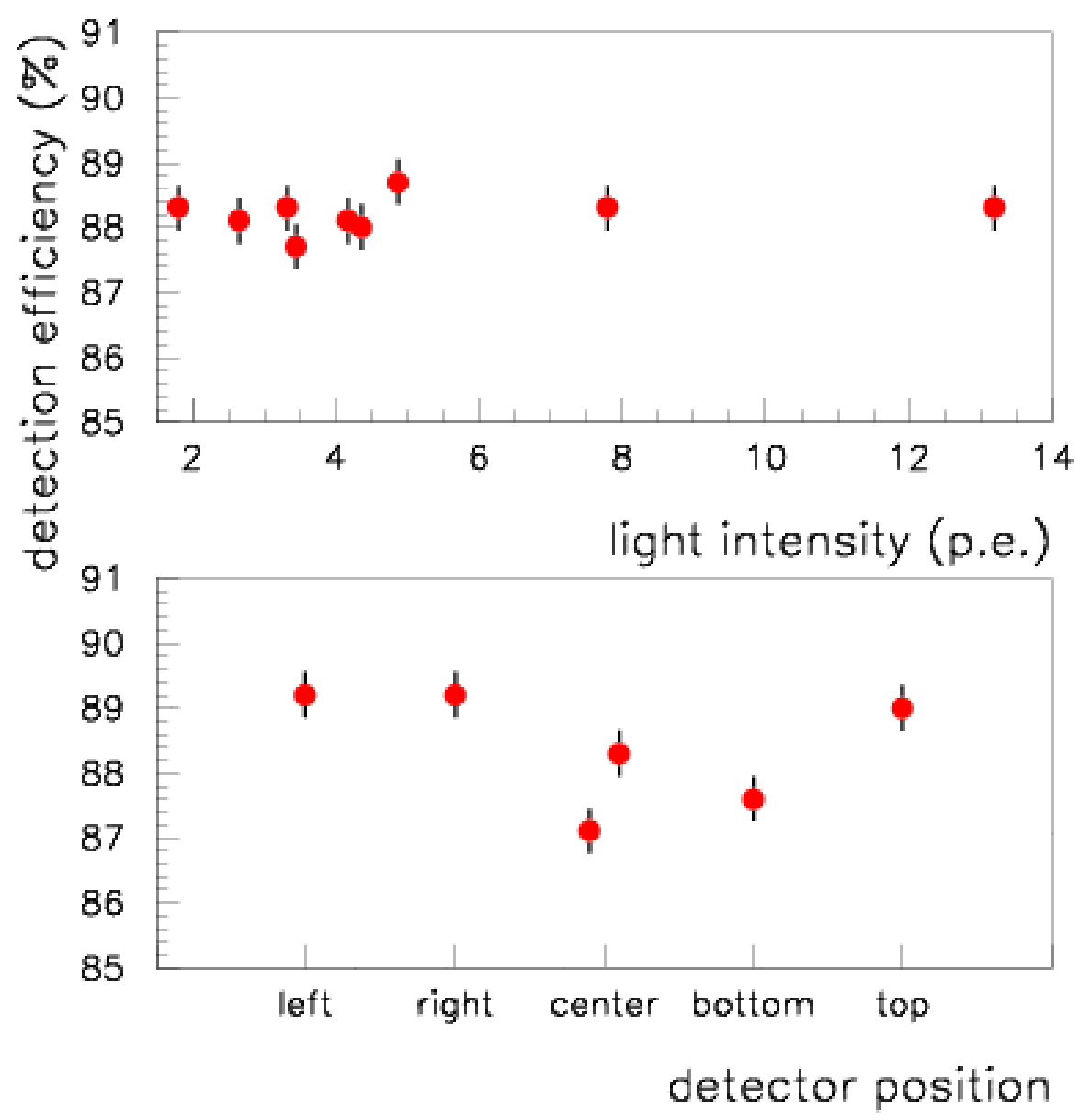

$>$ corrections to the binary data:

- pixel clustering (charge sharing) adjacent fired pixels are clustered together, assumed to originate from only one p.e.

- pixel clustering over-correction account for probability to have adjacent hits (function of light intensity and light profile) -missing bump bonds

$>$ backpulse uncertainties not incl.

$>$ stable photoelectron detection efficiency: $\sim \mathbf{8 8 \%}$ 


\section{Dark Counts and Ion Feed Back}

dark counts:

-thermal electron emission from photo cathode produces background signal

- average rate: $\sim 1 \mathrm{kHz} / \mathrm{cm}^{2}$ (depends on temperature and other experimental conditions) ion feed back (=afterpulses in PMTs):

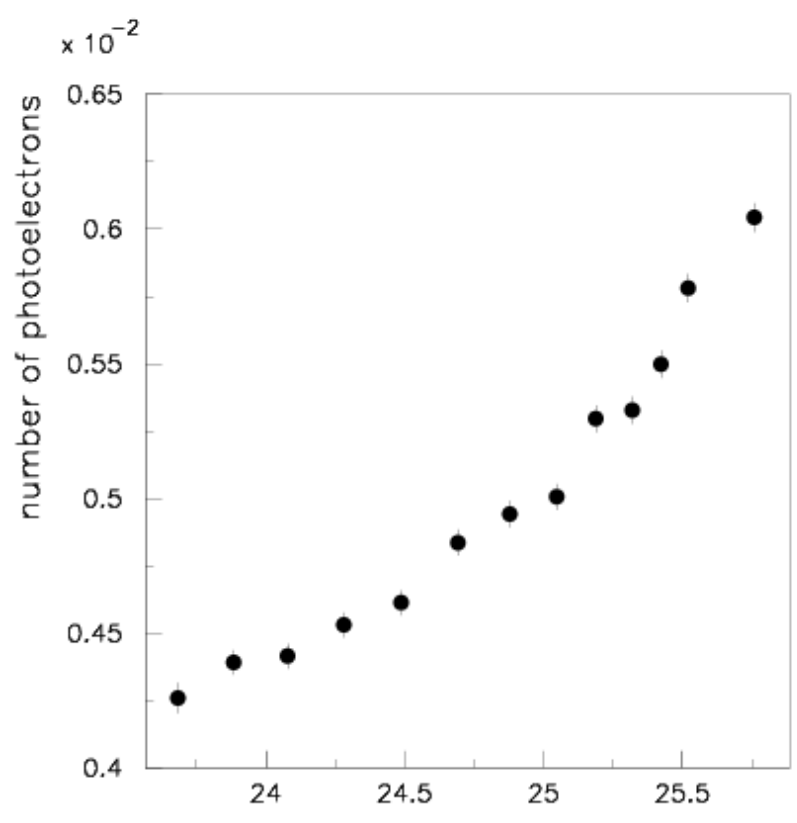

- ionized residual gas molecules hitting the cathode produce a cloud of photoelectrons

$>$ delayed ( $220 \mathrm{~ns})$ signal on one or (usually) more pixels - average rate: $<1 \%$ (depends on tube vacuum)

background comparable to multialkali PMTs 


\section{High Occupancy and Aging}

chip operated in LHCb mode: reduce 8192 channels to 1024 pulsed LED pulsed and DC LED only DC( $\sim 1 \%$ occup.)
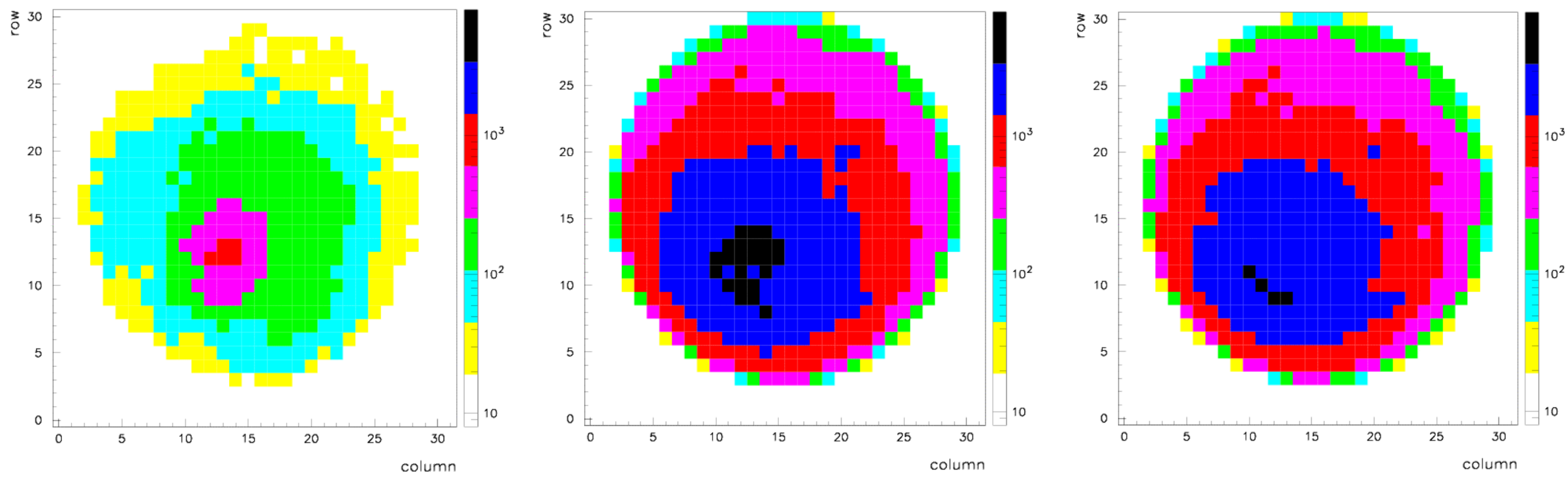

- no efficiency loss due to DC LED background signal

- aging test: simulate light exposure of 10 years LHC operation at $1 \%$ pixel occupancy: no efficiency (DE and QE) loss observed 


\section{Test-beam Measurements}
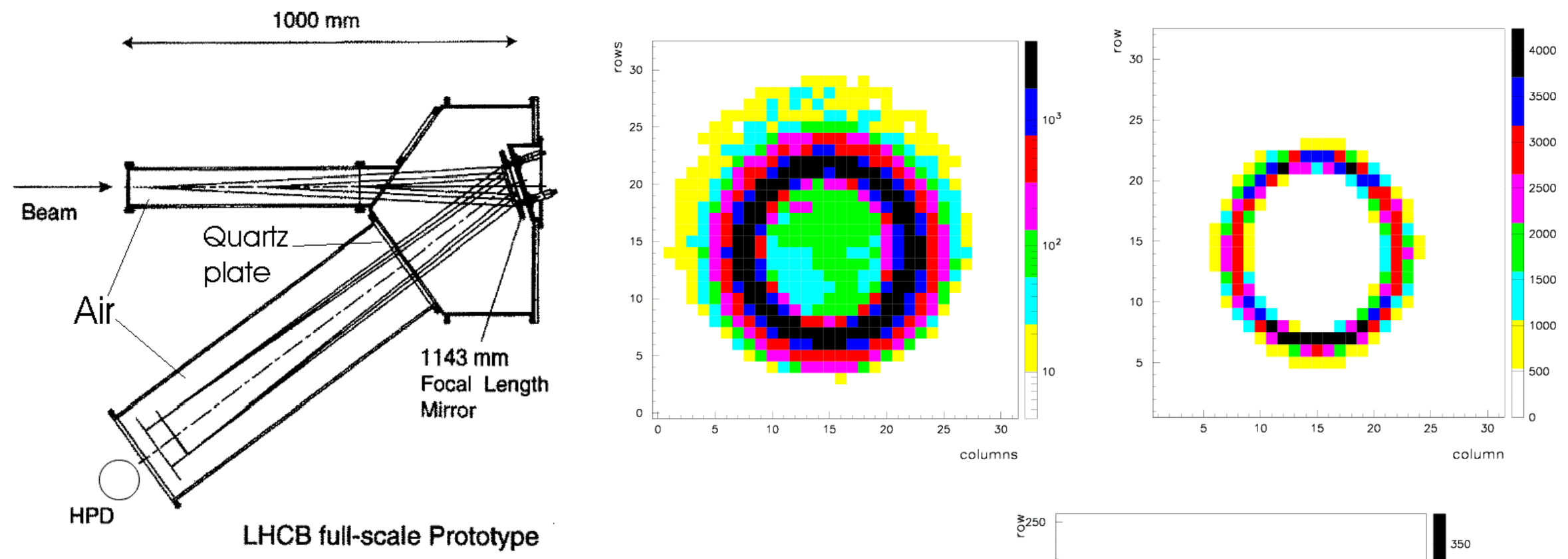

-10 GeV pions/electrons traversing through air

-Cherenkov rings focused on HPD $>$ all tests in agreement with LED results!

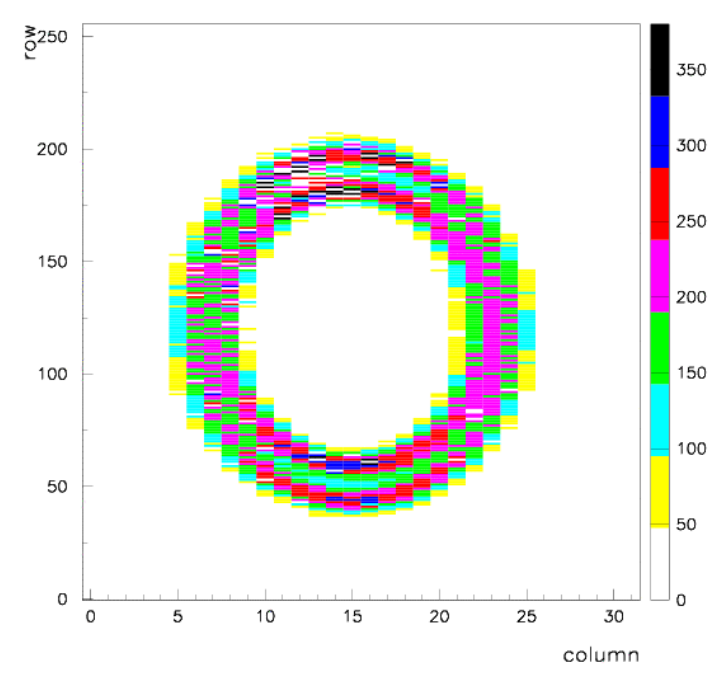




\section{$\underline{\text { Summary }}$}

Performance of two fully operational and functional $40 \mathrm{MHz}$ LHCb pixel HPDs studied:

- discriminating threshold ( 1700e $)$ and its spread $\left(\sim 250 \mathrm{e}^{-}\right)$ within specifications

- efficiency improvement with threshold adjustment demonstrated

- photoelectron detection efficiency stable $(\sim 88 \%)$ and above specification

- background due to dark counts and ion feed back small

- no performance degradation observed in operation with high pixel occupancy and after aging tests 